\title{
Morphometric analysis of the lumbar vertebrae in the Turkish population using three-dimensional computed tomography: correlation with sex, age, and height
}

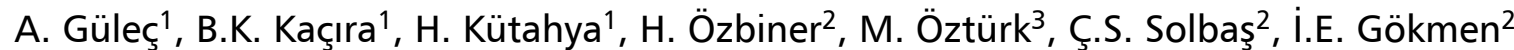 \\ ${ }^{1}$ Department of Trauma and Orthopaedics, Necmettin Erbakan University Meram Medical Faculty, Konya, Turkey \\ ${ }^{2}$ Department of Radiology, Necmettin Erbakan University Meram Medical Faculty, Konya, Turkey \\ ${ }^{3}$ Department of Radiology, Diyarbakır Children's Hospital, Diyarbakır, Turkey
}

[Received: 10 August 2016; Accepted: 16 December 2016]

Background: Morphometric measurements of lumbar vertebrae are different in European and Asian populations. Transpedicular screws are candidates for the ideal method to treat instability of lumbar vertebrae and provide very strong stabilisation. Our study reflects the variation of morphometric measurements of lumbar vertebrae in the Turkish population according to sex, age, and height. The aim of our study was to measure the transverse pedicle diameter (TPD), vertical pedicle diameter (VPD), pedicle axis length (PAL), and transverse pedicle angle (TPA) of the lumbar vertebrae, using three-dimensional computed tomography (3D-CT), and assess variations according to sex, age, and height.

Materials and methods: Prospective cohort, Therapeutic Level III, Urban Level III Trauma Centre. The study design adopted a morphometric analysis using 3D-CT of the lumbar vertebrae in the Turkish population, with variation in terms of sex, age, and height and comparison with previous studies. In 240 cases, measurements of TPC, VPD, PAL, and TPA with 3D-CT were performed on a total of 1200 lumbar vertebrae. The values at each lumbar level were compared in groups based on sex, age, and height. Results: The results of our study determined the normal values of TPD, VPD, PAL, and TPA of lumbar vertebrae in the Turkish population using 3D-CT. Additionally there were variations in TPD, VPD, and PAL according to sex, age, and height. TPA varied according to age, while no difference was found in terms of sex or height.

Conclusions: The morphometric measurements of lumbar vertebrae in the Turkish population are similar to western populations. Sex, age, and height are factors affecting reliable screw choice. (Folia Morphol 2017; 76, 3: 433-439)

Key words: lumbar pedicles, computed tomography, transpedicular screw fixation

\section{INTRODUCTION}

Used in the ideal treatment of spinal instability, pedicle screws provide very strong stabilisation. Though the use of radiological methods during the operation eases screw application, if they deviate from the expected path and exit the bone, they may cause neural tissue, visceral

Address for correspondence: Dr. M. Öztürk, Department of Radiology, Diyarbakır Children's Hospital, 21100, Diyarbakır, Turkey, e-mail: drmehmet2121@gmail.com 

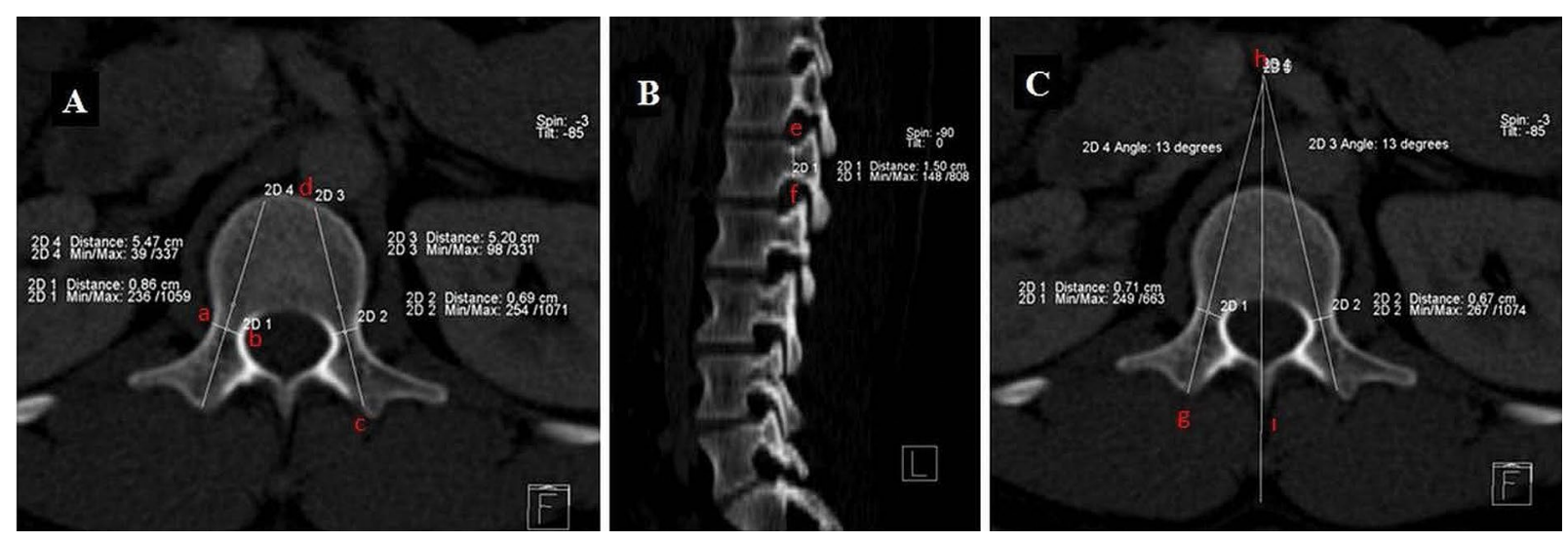

Figure 1. A, B, C. Measurement of A. Transverse pedicle diameter (a-b), pedicle axis length (c-d); B. Vertical pedicle diameter (e-f) and C. Transverse pedicle angle $(\mathrm{g}-\mathrm{h}-\mathrm{i})$ at the $\mathrm{L} 1$ vertebral level.

organ, or vascular injury. As a result, measurementbased morphology of the lumbar vertebrae, awareness of differences between levels, and choosing the screw appropriate for entry point angle and depth are important for an uncomplicated application [12]. Determination of factors controlling morphometric measurements affects appropriate choice of screw.

With the routine use of three dimensional computed tomography (3D-CT) in recent years, morphometric analysis studies assessing differences in lumbar vertebrae according to age and sex have been performed for different populations $[2,5,10]$.

The aim of our study is to transverse pedicle diameter (TPD), vertical pedicle diameter (VPD), pedicle axis length (PAL), and transverse pedicle angle (TPA) measurements on lumbar vertebrae using 3D-CT to evaluate variation according to sex, age, and height and research the effect on screw choice.

\section{MATERIALS AND METHODS}

This study included a total of 240 cases in which patients underwent 3D-CT for any reason on the lumbar vertebrae with no spinal pathology found, between January, 2008 and January, 2012 at the Radiology Department CT unit. The cases were divided into male and female groups; the two groups were then divided into four age groups of 30 females and 30 males as 20-29 years, 30-39 years, $40-49$ years, and $50-59$ years, and into four height groups as height $<160 \mathrm{~cm}(n=41)$, $160-169 \mathrm{~cm}(\mathrm{n}=96), 170-179 \mathrm{~cm}(\mathrm{n}=71)$, and $>$ $179 \mathrm{~cm}(\mathrm{n}=32)$. Variation in lumbar vertebral morphometric measurements was evaluated according to age, height, and sex. Cases with lumbar abnormalities (such as fracture, previous operation, tumoural lesion, or infection) identified on vertebral CT were excluded from the study.
Computed tomography investigation was performed with a 64-detector Siemens Sensation 64 (Erlangen, Germany) device. The detector configuration was $64 \times 0.6 \mathrm{~mm}$, gantry rotation speed: $0.5 \mathrm{~s}$, pitch value: 1 , tube voltage: $120 \mathrm{kV}$, and tube rated current: $160 \mathrm{~mA}$. For each measurement, multi-planar reconstructions of $1.5 \mathrm{~mm}$ slices were completed on a Leonardo workstation (Siemens Medical Solutions) and axial, coronal, and vertical reformatted images according to vertebral axis were created.

\section{Measurements}

From the obtained 3D images, the following measurements were made between the L1-L5 vertebral levels.

- TPD $=$ transverse pedicle diameter - the distance between both cortical walls of the pedicle in the transverse plane (Fig. 1A, distance from ' $a$ ' to ' $b$ ');

- PAL = pedicle axis length - the distance between the posterior cortex of the pedicle and vertebra corpus anterior along the pedicle axis (Fig. 1A, distance from ' $c$ ' to ' $d$ ');

- VPD $=$ vertical pedicle diameter - the distance between both cortical walls of the pedicle in the vertical plane (Fig. 1B, distance from ' $e$ ' to ' $f$ ');

- TPA $=$ transverse pedicle angle - the angle between the pedicle axis and midline in the transverse plane (Fig. 1C, angle between ' $g$ ' and ' $h$ ' and ' $i$ ').

\section{Statistical analysis}

All TPD, VPD, PAL, and TPA measurements were completed on the right and left. For right and left measurements, for those without statistical significance the right was used, for those with statistical significance the lower value was used. In situations where parametric data had normal distribution, to determine right-left and sex effects and test the mean of two 


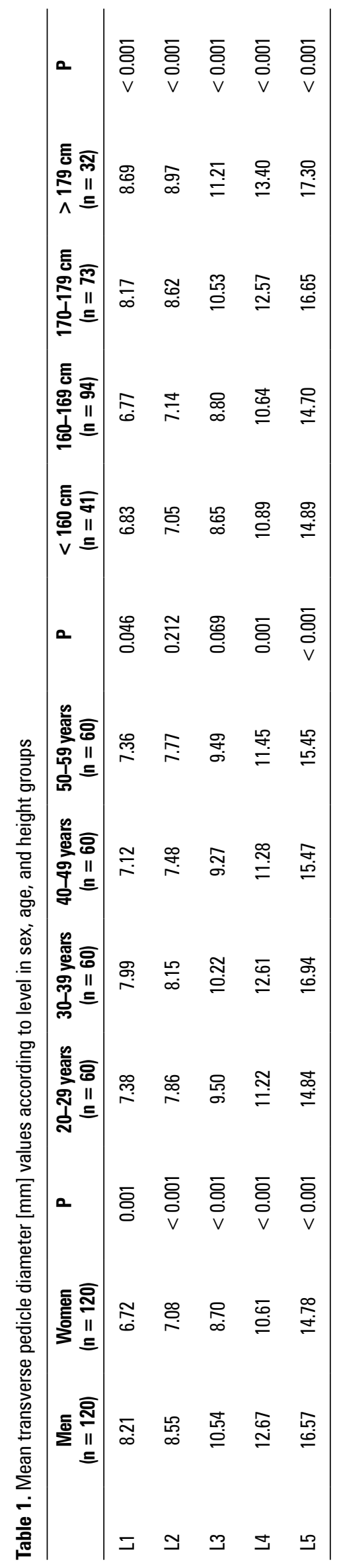

independent groups, the Student's t-test was used. To determine right-left and sex effects for parameters without normal distribution, the non-parametric independent two-sample group test of the Mann-Whitney $\mathrm{U}$ test was used. To evaluate the quadruple groups of age and height, for parameters with normal distribution, the one-way variance analysis was completed. For significant differences between the groups, the Duncan method was used for pairwise comparisons, and $p<0.05$ was accepted as significant. To evaluate the differences between groups of parameters with non-normal distribution, the Kruskal-Wallis method of k-independent group test was used. Significant group differences were determined with pairwise comparisons between groups with the Mann-Whitney $\mathrm{U}$ test at $p<0.05$ level.

\section{RESULTS}

TPD

Mean TPD values according to level in sex, age, and height groups are summarised in Table 1. According to sex, all levels were statistically significant, with men having higher values. Statistically significant differences between age-groups were only found at the L4 and $L 5$ levels, with the 30-39 age-group having the highest values. In terms of height groups, all levels were significant with the $170-179 \mathrm{~cm}$ and $>179 \mathrm{~cm}$ groups similar with highest values.

VPD

The mean VPD values according to level in sex, age, and height groups are summarised in Table 2. According to sex, all levels were statistically significantly different, with men having higher values. In terms of age groups, all levels were statistically significantly different, with the highest value in the 30-39 age group. Between height groups, at all levels apart from $L 5$, the $170-179 \mathrm{~cm}$ and $>179 \mathrm{~cm}$ groups were similar, with the highest values.

PAL

The mean PAL values according to level in sex, age, and height groups are summarised in Table 3 . In terms of sex, there were statistically significant differences at all levels, with males having higher values. Between age groups, there were statistically significant differences at all levels, with the 30-39 age group having highest values. In terms of height groups, at all levels the $170-179 \mathrm{~cm}$ and $>179 \mathrm{~cm}$ groups were similar, with the highest values. 

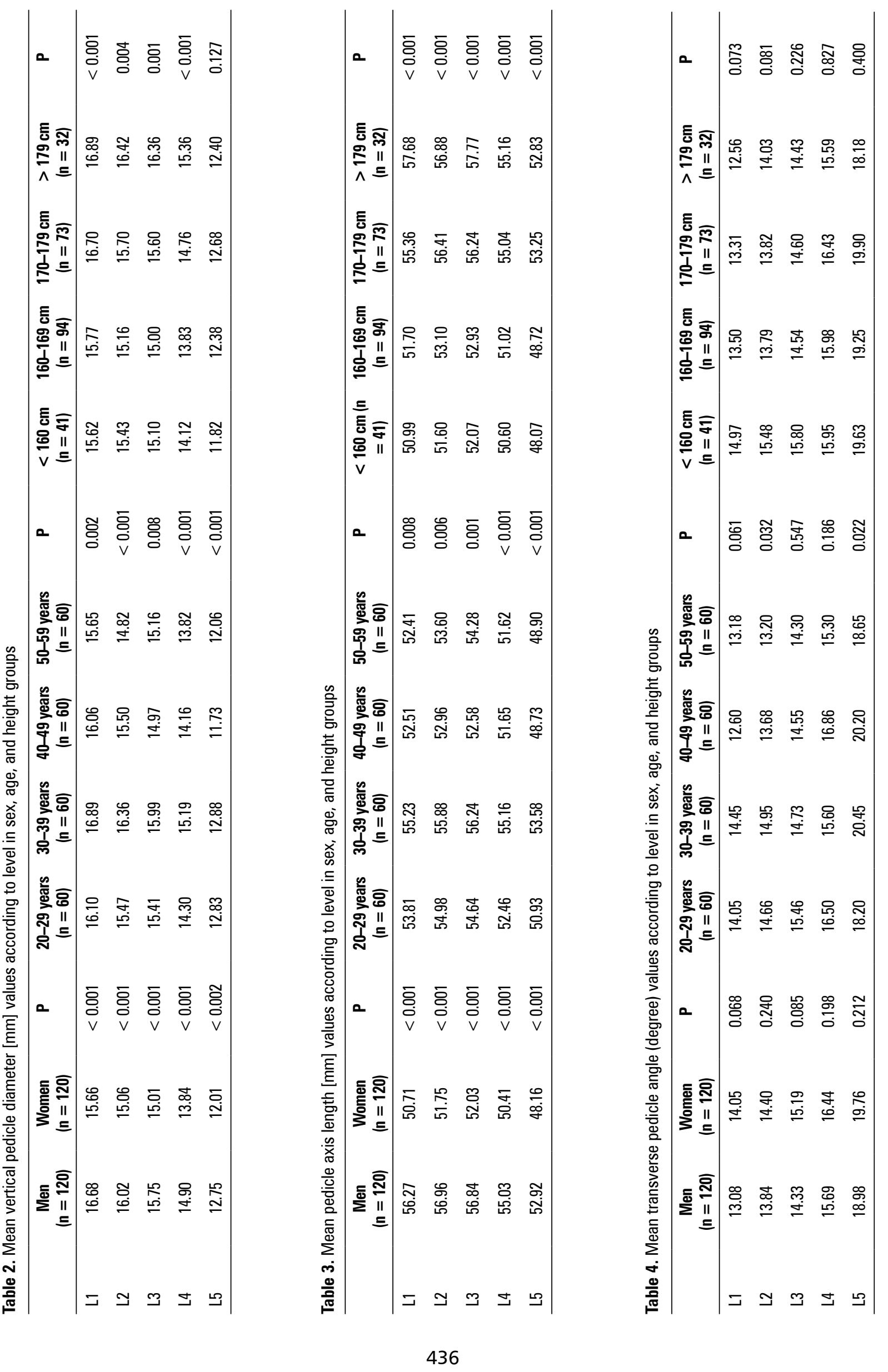
TPA

Mean TPA values according to level in sex, age, and height groups are summarised in Table 4. At all levels there was no difference in terms of sex. In terms of age groups, only at L2 and L5 levels there was a statistically significant difference, with the highest values for the 30-39 group. There were no differences identified for height groups at all levels.

\section{DISCUSSION}

This study performed morphometric analysis on lumbar vertebrae using the 3D-CT method in the Turkish population and determined important results. The first result, that normal values of lumbar vertebrae in the Turkish population reflect variations according to sex, age, and height, is important. The second result is that TPD, VPD, and PAL vary according to sex, age, and height. TPA varies according to age, with no variation according to sex or height. The third result is that the values are similar to those from western populations.

\section{TPD}

To insert the screw appropriately in the pedicle without damaging medial and lateral cortices, the most important parameter to determine size is TPD. In two previous studies of 51 cases in western populations and 40 cases in China, TPD was reported to be higher in men $[7,11]$. Another study identified differences according to sex only at L3 and L5 levels [8]. A study assessing 49 cases with 3D-CT in the Pakistani population found that males had higher values than females at all lumbar levels [2]. A cadaver study evaluating a total of 75 cases by CT without sex differentiation in Egypt found TPD values were $6.8 \pm$ $\pm 1.9 \mathrm{~mm}$ at $\mathrm{L} 1$ and $18.9 \pm 2.1$ at L5, with a direct increase observed from L1 to L5 [10]. A study assessing a total of 50 cases, 25 male and 25 female in the Iranian population found that TPD values were higher at all levels in men [9]. The results of measuring 503 cadavers from the American population identified a direct increase from L1 to L5, with males having higher values at all levels [13]. The TPD values determined in our study are similar to those from western populations. As a result, screws currently produced for western populations may be used for the Turkish population. However, patient sex must also be considered for screw choice.

A study of TPD variation according to age using direct X-ray on a total of 540 cases in the Arab population found the widest diameter in females from 10 to
19 years and in males from 40 to 49 years [3]. A study of 503 cadavers in the American population found an increase in TPD at all levels, with age measurements made without reference to sex [13]. This study did not differentiate age groups according to sex and found the highest values were in the 30-39 age group. TPD values did not show a simple linear increase or decrease between age groups. Though the highest TPD value was in the 30-39 age group, at L1 level in the other groups, the difference was less than $1 \mathrm{~mm}$, which is not clinically significant. However, at the L4 and $\mathrm{L} 5$ levels, the difference was more than $1 \mathrm{~mm}$. As a result, especially in the 30-39 age group, the differences at the L4 and L5 levels should be noted during screw choice.

Variation in TPD according to height was previously shown to increase with the increase in height at all levels as a result of the 503 cadaver American population study without reference to sex [13]. Similarly, in our study, TPD values showed a proportional increase with height, with the highest values for all levels in the 170-179 cm and $>179 \mathrm{~cm}$ height groups. The difference in mean TPD values between heights above $170 \mathrm{~cm}$ and those below was identified as $2 \mathrm{~mm}$. This result shows that patient height should be noted when choosing screws.

\section{VPD}

In two different studies of VPD with CT and X-ray in western populations, at other lumbar levels apart from L5, VPD was found to be higher than TPD [14]. A study of the Chinese population found that VPD was higher than TPD, at all levels, with a direct increase identified from L1 to L5, contrary to the other studies $[7,11]$. A study evaluating 49 cases with 3D-CT in the Pakistani population found it was higher than TPD at all lumbar levels, and there was a reduction toward L5, while it was higher in males than in females [2]. The American population study of 503 cadavers found a direct reduction from $L 1$ to $L 5$, with higher values for males at all levels [13]. A previous study of the Turkish population measured only L5 levels of 40 cases and found VPD was lower than TPD [4]. In our study, 3D-CT measurements were performed for all levels in the Turkish population, and it provides important data due to the larger case number. The values obtained were similar to western populations, with value higher than TPD at all levels apart from L5 and a reduction from L1 to L5. It varied with sex and was higher in males. 
In the Arab population study of 540 cases evaluating the change in VPD according to age using direct $X$-ray, for women there was a reduction from the 30-39.9 to 40-49.9 age group at all levels apart from L1, with an increase observed from the 40-49.9 age group to the $50+$ age group. In men, a reduction was observed from the 20-29.9 age group to the 30-39.9 age group [3]. The American population study of 503 cadavers did not reference sex during measurements and identified an increase in VPD with age at all levels [13]. At all levels in our study, the highest value was in the 30-39 age group, with the lowest values generally observed in the 50-59 age group. However, as the differences between the groups at every level were around $1 \mathrm{~mm}$, this difference was not accepted as clinically significant.

Measurements of the variation in VPD according to height in the American population cadaver study without reference to sex identified an increase in VPD at all levels as height increased [13]. In our study, the variation in VPD values according to height was statistically significant at all levels apart from L5, with the highest values in the $170-179 \mathrm{~cm}$ and $>179 \mathrm{~cm}$ groups and the lowest values in the $<160 \mathrm{~cm}$ and $160-169 \mathrm{~cm}$ groups. As height increased, VPD increased. In height groups above and below $170 \mathrm{~cm}$, there was a difference of $2 \mathrm{~mm}$ observed in VPD values. In conclusion, VPD varies according to height.

\section{PAL}

Knowing the PAL value before operation is very important to prevent anterior cortex perforation. A CT study of PAL in western populations measured L2 as $53.3 \pm 0.6 \mathrm{~mm}, \mathrm{~L} 353.3 \pm 3.2 \mathrm{~mm}, \mathrm{~L} 455.4 \pm$ $\pm 3.0 \mathrm{~mm}$, and L5 $52.0 \pm 4.5 \mathrm{~mm}$ in males, and L2 as $49.7 \pm 4.7 \mathrm{~mm}$, L3 $47.0 \pm 3.4 \mathrm{~mm}$, L4 $50.2 \pm$ $\pm 4.7 \mathrm{~mm}$, and $\mathrm{L} 546.0 \pm 5.7 \mathrm{~mm}$ in females. In both sexes, the highest value was at $L 4$, while the smallest value was at L5 [11]. A 50 patients CT study of the Indian population without reference to sex found PAL values were L1 $47 \pm 3.39 \mathrm{~mm}, \mathrm{~L} 249.03 \pm 3.39 \mathrm{~mm}$, L3 $47.21 \pm 3.95 \mathrm{~mm}$, L4 $47.48 \pm 5.38 \mathrm{~mm}$ and $L 548.91 \pm 4.42 \mathrm{~mm}$, with the highest value for $L 2$ and the lowest value for L1 [1]. A study of 50 males and 50 females aged from 20 to 40 years used magnetic resonance imaging to measure only the L1 level and found values were L1 $47.11 \pm 6.14 \mathrm{~mm}$ for males and L1 $40.61 \pm 6.31 \mathrm{~mm}$ for females [6]. The Egyptian study using $\mathrm{CT}$ to evaluate a total of
75 cases and cadavers without reference to sex found PAL values were $\mathrm{L} 151 \pm 4.9 \mathrm{~mm}$ and $\mathrm{L} 548 \pm 5.1 \mathrm{~mm}$ with a direct reduction from L1 to L5 [10].

The Iranian population study evaluating 25 males and 25 females CT found PAL values were L1 49.88 $\mathrm{mm}$ and $45.08 \mathrm{~mm}$ and $\mathrm{L} 550.2 \mathrm{~mm}$ and $46.4 \mathrm{~mm}$ for males and females, respectively, with male values higher at all levels compared to females and a direct reduction from L1 to L5 [9]. In our study, PAL values varied according to sex at all levels, with males having higher measurements. The data showed that L1, L2 and L3 were close to each other with a reduction in L4 and L5. In both sexes, the largest values were at L3 (male $56.84 \mathrm{~mm}$, female $52.03 \mathrm{~mm}$ ), with the smallest values at $L 5$ (male $52.92 \mathrm{~mm}$, female $48.16 \mathrm{~mm}$ ). The values were similar to data from western populations.

When the variation in PAL according to age was evaluated in our study, the highest values at all levels were in the 30-39 age group. The difference between the lowest and highest PAL values among the groups was 2-4 mm, which is statistically significant. However, as the variation interval for screws is $5 \mathrm{~mm}$, this result is not accepted as clinically significant.

In our study, the variation in PAL according to height was evaluated. PAL showed a proportional increase with height. In groups with heights of $170 \mathrm{~cm}$ and below, there was a $5 \mathrm{~mm}$ difference in mean PAL values. In conclusion, the variation of PAL according to height, affects the variation interval of screws and their choice.

\section{TPA}

The TPA is a very important parameter to determine the insertion direction of screws. A CT study related to TPA in western populations did not find a statistical difference between the sexes but did find a direct increase from L2 to L5 [2]. A study using CT and $X$-rays without referencing sex measured TPA as L1: 10.9, L2: 12.0, L3: 14.4, L4: 17.7, and L5: 29.8 degrees [6]. The CT study of 50 cases from the Indian population without reference to sex found TPA was $10.90 \pm 3.13$ at L1 and $24.75 \pm 3.83$ at L5, similar to western populations [4]. The study evaluating 49 Pakistani cases with 3D-CT at all lumbar levels found L1 was 13.4, and L5 was 21.6 degrees, with an increase identified toward L5 [2]. The Egyptian $\mathrm{CT}$ and cadaver study of 75 cases without reference to sex found TPA values were $15 \pm 2.7$ at L1 and $30 \pm 3.6$ degrees at $L 5$, with a direct increase from 
L1 to L5 [10]. The 50-case Iranian study of 25 males and 25 females using CT found TPA values were 16.76 and 15.88 at L1 and 23.68 and 24.56 at L5 for males and females, respectively, with a direct increase from L1 to L5 in both sexes [9]. This study did not find differences in TPA values according to sex at all levels. There was a convergent increase observed from L1 to L5.

In our study, the variation in TPA was assessed according to age. Though there was a statistical difference between the groups at L2 and L5 levels, this was not accepted as clinically significant.

Our study evaluated the change in TPD according to height. There was no change in TPA values identified according to height.

There are some limitations to our study. The oldest age interval included in our study was 50-59 years, and those over 60 years of age, when degenerative lumbar disease is commonly observed, were excluded.

\section{CONCLUSIONS}

In conclusion, our study researched detailed morphometric properties at five different lumbar vertebral levels in 240 cases and the variation of these properties according to sex, age, and height. All of these factors are important for screw choice, and we believe the general trends in our results must be noted to prevent complications that may develop during operations.

\section{REFERENCES}

1. Acharya S, Dorje T, Srivastava A. Lower dorsal and lumbar pedicle morphometry in Indian population: a study of four hundred fifty vertebrae. Spine (Phila Pa 1976). 2010; 35(10): E378-E384, doi: 10.1097/BRS.0b013e3181cb7f2b, indexed in Pubmed: 20431473.

2. Alam MM, Waqas M, Shallwani $\mathrm{H}$, et al. Lumbar morphometry: a study of lumbar vertebrae from a pakistani population using computed tomography scans. Asian Spine J. 2014; 8(4): 421-426, doi: 10.4184/asj.2014.8.4.421, indexed in Pubmed: 25187858.
3. Amonoo-Kuofi HS. Age-related variations in the horizontal and vertical diameters of the pedicles of the lumbar spine. J Anat. 1995; 186: 321-328, indexed in Pubmed: 7649830.

4. Azar N, Akman YE, Yalçınkaya M, et al. L5 vertebral morphometric characteristics of Turkish society. J Turk Spinal Surg. 2008; 19: 373-380.

5. Bing Li, Baoguo J, Zhongguo Fu, et al. Accurate determination of isthmus of lumbar pedicle: a morphometric study using reformatted computed tomographic images. Spine. 2004; 29(21): 2438-2444, doi: 10.1097/01. brs.0000144355.50660.65.

6. Çapar B, Karagüven D, Benli IT, et al. Morphometric analysis of thoracolumbar pedicle dimensions of the adolescent and adult age groups. J Turkish Spinal Surg. 2012; 23: 19-26.

7. Hou S, Hu R, Shi Y. Pedicle morphology of the lower thoracic and lumbar spine in a Chinese population. Spine (Phila Pa 1976). 1993; 18(13): 1850-1855, indexed in Pubmed: 8235871.

8. Kim NH, Lee HM, Chung $\mathrm{IH}$, et al. Morphometric study of the pedicles of thoracic and lumbar vertebrae in Koreans. Spine. 1994; 19(12): 1390-1394, indexed in Pubmed: 8066521.

9. Lotfinia I, Haddadi K, Sayyahmelli S. Computed tomographic evaluation of pedicle dimension and lumbar spinal canal. Neurosurg Quar. 2010; 20(3): 194-198, doi: 10.1097/wnq.0b013e3181 eb284a.

10. Mohamed A. M, Adel Saad B, Mohey EE. Morphological measurements of lumbar pedicles in Egyptian population using computerized tomography and cadaver direct caliber measurements. Egypt J Radiol Nucl Med. 2010; 41(4): 475-481, doi: 10.1016/j.ejrnm.2010.10.002.

11. Olsewski JM, Simmons EH, Kallen FC, et al. Morphometry of the lumbar spine: anatomical perspectives related to transpedicular fixation. J Bone Joint Surg Am. 1990; 72(4): 541-549, indexed in Pubmed: 2139030.

12. West JL, Ogilvie JW, Bradford DS. Complications of the variable screw plate pedicle screw fixation. Spine. 1991; 16(5): 576-579, indexed in Pubmed: 2053001.

13. Yu CC, Yuh RT, Bajwa NS, et al. Pedicle morphometry of lumbar vertebrae: male, taller, and heavier specimens have bigger pedicles. Spine. 2015; 40(21): 1639-1646, doi: 10.1097/BRS.0000000000001086, indexed in Pubmed: 26244405.

14. Zindrick MR, Wiltse LL, Doornik A, et al. Analysis of the morphometric characteristics of the thoracic and lumbar pedicles. Spine. 1987; 12(2): 160-166, indexed in Pubmed: 3589807. 